\title{
Linear Ascending Metrological Algorithm
}

\author{
M. R. Perelshtein $\odot,{ }^{1,2,3,{ }^{*}}$ N. S. Kirsanov ${ }^{1},{ }^{1,2,3,4, *}$ V. V. Zemlyanov, ${ }^{1,2}$ A. V. Lebedev, ${ }^{2}$ G. Blatter, ${ }^{5}$ \\ V. M. Vinokur ${ }^{1}{ }^{1}$ and G. B. Lesovik ${ }^{1,2}$ \\ ${ }^{1}$ Terra Quantum AG, St. Gallerstrasse 16A, 9400 Rorschach, Switzerland \\ ${ }^{2}$ Moscow Institute of Physics and Technology, 141700, Institutskii Pereulok 9, Dolgoprudny, Moscow District, Russian Federation \\ ${ }^{3}$ QTF Centre of Excellence, Department of Applied Physics, Aalto University School of Science, P.O. Box 15100, FI-00076 Aalto, Finland \\ ${ }^{4}$ Consortium for Advanced Science and Engineering (CASE), University of Chicago, 5801 S Ellis Avenue, Chicago, Illinois 60637, USA \\ ${ }^{5}$ Theoretische Physik, Wolfgang-Pauli-Strasse 27, ETH Zürich, CH-8093 Zürich, Switzerland
}

(Received 22 July 2020; accepted 4 January 2021; published 19 March 2021)

\begin{abstract}
The ubiquitous presence of shot noise sets a fundamental limit to the measurement precision in classical metrology. Recent advances in quantum devices and novel quantum algorithms utilizing interference effects are opening new routes for overcoming the detrimental noise tyranny. However, further progress is limited by the restricted capability of existing algorithms to account for the decoherence pervading experimental implementations. Here, adopting a systematic approach to the evaluation of effectiveness of metrological procedures, we devise the Linear Ascending Metrological Algorithm (LAMA), which offers a remarkable increase in precision in the demanding situation where a decohering quantum system is used to measure a continuously distributed variable. We introduce our protocol in the context of magnetic field measurements, assuming superconducting transmon devices as sensors operated in a qudit mode. Our findings demonstrate a quantum-metrological procedure capable of mitigating detrimental dephasing and relaxation effects.
\end{abstract}

DOI: 10.1103/PhysRevResearch.3.013257

\section{INTRODUCTION}

Shot noise sets a fundamental limit to measurement precision, which is often referred to as "noise tyranny." Quantum algorithms utilizing interference effects offer new opportunities to overcome it [1-7]. However, existing algorithms cannot account for decoherence-controlled experimental conditions [8]. Our work develops a systematic approach in the evaluation of the effectiveness of metrological procedures and the seeking of efficient solutions. To that end, we devise the Linear Ascending Metrological Algorithm (LAMA), applicable to and guaranteeing an enhanced precision in the case where a decoherent quantum system is used to measure a continuously distributed variable. We introduce our protocol in the context of magnetic field measurements, assuming superconducting transmon devices as sensors operated in a qudit mode [9].

Phase estimation protocols first appeared in abstract quantum algorithms, where they served in estimating the phases of a unitary operator's eigenvectors, and soon found practical applications in quantum metrology [10-16]. Among many implementations, the Kitaev [17,18] and Fourier-transform [19] algorithms, in combination with superconducting transmon circuits [20] utilized as sensors, proved most efficient in

\footnotetext{
${ }^{*}$ These authors contributed equally to this work.
}

Published by the American Physical Society under the terms of the Creative Commons Attribution 4.0 International license. Further distribution of this work must maintain attribution to the author(s) and the published article's title, journal citation, and DOI. magnetometry. The basic concept of a magnetic field sensor based on a spin interacting with the field has evolved into experimentally realizable devices based on charge and flux qudits [8,21-23]. However, the standard quantummetrological protocols concede their optimal precision in the most relevant situation where the field is continuously distributed and the quantum sensor suffers from decoherence. Here, we devise a simple and practical protocol for quditsLAMA - allowing us to appreciably enhance the efficiency of the measurement over the standard Fourier-transform and Kitaev algorithms. Distinct from the latter, our protocol benefits from a maximum average spin component perpendicular to the field and takes advantage of a linear stepwise increase in the Ramsey delay-time interval. Throughout our analysis, the operation of the different metrological algorithms will be addressed in the context of their qutrit [24,25] (base 3) transmon realization, as, unlike the example of a qubit (base 2) realization, this allows us to demonstrate the new algorithm's full potential.

\section{GENERAL PHASE-SENSITIVE PROTOCOL}

We begin with a description of the general base- $d$ phasesensitive metrological procedure employing a sequential strategy, with each step following the preparation-exposurereadout (PER) logic. The procedure is aimed at the measurement of a constant magnetic field $H$. We work with the computational basis states $|0\rangle,|1\rangle, \ldots,|d-1\rangle$ corresponding to different magnetic components $\mathfrak{M}_{Z}^{d}$ with respect to the field direction ( $Z$ axis): For instance, in the qutrit case, the basis vectors $|0\rangle,|1\rangle$, and $|2\rangle$ correspond to $\mathfrak{M}_{Z}^{3}=-\mu, 0,+\mu$, 
respectively, where $\mu$ denotes the magnetic moment of the artificial atom [8], which serves as a coupling constant and which is known a priori. The $i$ th step of the general procedure involves a Ramsey interference with delay time $t_{i}$ and is described as follows:

$\mathbf{P}$ The qudit is prepared in a defined initial state $\left|\psi_{(i)}^{0}\right\rangle$; this is experimentally realized by applying a suitable rf pulse to the qudit ground state [9].

E The qudit interacts with the external magnetic field $H$ during a time $t_{i}:\left|\psi_{(i)}^{0}\right\rangle \rightarrow\left|\psi_{(i)}^{t_{i}}\right\rangle$. The field changes the phases inside the state vector such that the basis state $|k\rangle$ $(k=\{0,1, \ldots, d-1\})$ transforms into $e^{i k \omega t_{i}}|k\rangle$, where $\omega=$ $\mu H / \hbar$ is the reduced magnetic field. We omit a common phase factor; see Appendix A for details on the transmon phase accumulation dynamics.

$\mathbf{R}$ The qudit is subjected to a readout operation (unitary evolution) $\hat{U}_{(i)}^{r}$ generated by another rf pulse [9]. The information about the field value is subsequently extracted through the final state's single-shot projective measurement in the computational basis.

The probability of finding the qudit in the state $\left|\xi_{i}\right\rangle\left(\xi_{i}=\right.$ $\{0,1, \ldots, d-1\})$ at the end of the $i$ th step is

$$
P\left(\xi_{i} \mid \omega, t_{i}, \mathbf{s}_{i}\right)=\left|\left\langle\xi_{i}\left|\hat{U}_{(i)}^{r}\right| \psi_{(i)}^{t_{i}}\right\rangle\right|^{2},
$$

where $\mathbf{s}_{i}$ is the array of parameters determining the initial state and the readout operation; see Appendix B. Throughout the metrological procedure, or "learning" process, our knowledge about the field is reflected in the probability distribution $\mathcal{P}^{n}\left(\omega \mid\left\{\xi_{i}, t_{i}, \mathbf{s}_{i}\right\}_{i=1}^{n}\right)$, where $n$ indicates the number of conducted PER steps. This distribution is updated in accord with Bayes's theorem via the recurrence

$$
\begin{aligned}
& \mathcal{P}^{n}\left(\omega \mid\left\{\xi_{i}, t_{i}, \mathbf{s}_{i}\right\}_{i=1}^{n}\right) \\
& \quad=\mathcal{P}^{n-1}\left(\omega \mid\left\{\xi_{i}, t_{i}, \mathbf{s}_{i}\right\}_{i=1}^{n-1}\right) P\left(\xi_{n} \mid \omega, t_{n}, \mathbf{s}_{n}\right) \mathcal{N}_{n},
\end{aligned}
$$

where $\mathcal{N}_{n}$ is a normalization factor; for ease of presentation (see Appendix F), we will assume that the initial field distribution is Gaussian with zero mean, $\mathcal{P}^{0}(\omega \mid \varnothing)=N\left(0, \sigma^{2}\right)$. As shown below, the initial field uncertainty $\delta \omega_{0}=\sigma$ determines the difficulty of further refinement: The smaller $\delta \omega_{0}$ is, the harder it is to achieve better precision.

\section{EFFICIENT PROCEDURE}

In an efficient metrological procedure, a measurement step strongly reduces the uncertainty with regard to the possible field values. This uncertainty is reflected in the Shannon entropy associated with $\mathcal{P}^{n}$,

$$
\begin{aligned}
& S^{n}\left(\left\{\xi_{i}, t_{i}, \mathbf{s}_{i}\right\}_{i=1}^{n}\right) \\
& \quad=-\int \mathcal{P}^{n}\left(\omega^{\prime} \mid\left\{\xi_{i}, t_{i}, \mathbf{s}_{i}\right\}_{i=1}^{n}\right) \ln \mathcal{P}^{n}\left(\omega^{\prime} \mid\left\{\xi_{i}, t_{i}, \mathbf{s}_{i}\right\}_{i=1}^{n}\right) d \omega^{\prime} .
\end{aligned}
$$

The decrease $\Delta I^{n}=S^{n-1}-S^{n}$ in entropy then provides us with the information gain in the $n$th measurement step. The optimal procedure yielding the maximum information gain in the $(n+1)$ st step requires the best choice of the parameters $t_{n+1}$ and $\mathbf{s}_{n+1}$. Since the values of parameters are required before the step is executed, the optimization has to be done by maximizing an estimate of the prospective information gain. This estimate is taken as the information gain after the $(n+1)$ st step averaged over all possible outcomes $\tilde{\xi}_{n+1}$ of this step.

$$
\begin{aligned}
& \left\langle\Delta I^{n+1}\left(\left\{\xi_{i}, t_{i}, \mathbf{s}_{i}\right\}_{i=1}^{n},\left\{\tilde{\xi}_{n+1}, t, \mathbf{s}\right\}\right)\right\rangle \\
& \quad=S^{n}\left(\left\{\xi_{i}, t_{i}, \mathbf{s}_{i}\right\}_{i=1}^{n}\right)-\left\langle S^{n+1}\left(\left\{\xi_{i}, t_{i}, \mathbf{s}_{i}\right\}_{i=1}^{n},\left\{\tilde{\xi}_{n+1}, t, \mathbf{s}\right\}\right)\right\rangle,
\end{aligned}
$$

where

$$
\begin{aligned}
& \left\langle S^{n+1}\left(\left\{\xi_{i}, t_{i}, \mathbf{s}_{i}\right\}_{i=1}^{n},\left\{\tilde{\xi}_{n+1}, t, \mathbf{s}\right\}\right)\right\rangle \\
& =\sum_{\tilde{\xi}_{n+1}=0}^{d-1} \int S^{n+1}\left(\left\{\xi_{i}, t_{i}, \mathbf{s}_{i}\right\}_{i=1}^{n},\left\{\tilde{\xi}_{n+1}, t, \mathbf{s}\right\}\right) \\
& \quad \times P\left(\tilde{\xi}_{n+1} \mid \omega^{\prime}, t, \mathbf{s}\right) \mathcal{P}^{n}\left(\omega^{\prime} \mid\left\{\xi_{i}, t_{i}, \mathbf{s}_{i}\right\}_{i=1}^{n}\right) d \omega^{\prime} .
\end{aligned}
$$

The optimal choice of parameters $\{t, \mathbf{s}\}=\left\{t_{n+1}, \mathbf{s}_{n+1}\right\}$ is dictated by the condition of maximizing the average gain $\left\langle\Delta I^{n+1}\left(\left\{\xi_{i}, t_{i}, \mathbf{s}_{i}\right\}_{i=1}^{n},\left\{\tilde{\xi}_{n+1}, t, \mathbf{s}\right\}\right)\right\rangle$. In other words, the analytical expression for the average information gain serves as a prognosis for the upcoming information gain, and its maximization yields the most beneficial choice of parameters.

In order to find the optimal choice for $\mathbf{s}_{1}$ defining the preparation and readout gates in the first step, we maximize the expected information gain $\left\langle\Delta I^{1}\left(\tilde{\xi}_{1}, t, \mathbf{s}\right)\right\rangle$ for every possible value of the Ramsey delay time $t$. Focusing on the case of a continuously distributed field (i.e., $\omega$ can take any value from a certain interval $\left.\left[\omega_{\min }, \omega_{\max }\right]\right)$ measured with a qutrit sensor, a numerical analysis (see Appendix B) shows that, intriguingly, for any $t$ the optimal initial state preparation requires a maximum modulus of the spin projection into the $X Y$ plane, perpendicular to the field vector $\left\langle J_{X Y}\right\rangle=\sqrt{\left\langle\hat{J}_{X}\right\rangle^{2}+\left\langle\hat{J}_{Y}\right\rangle^{2}}$ (here, $\hat{J}_{X}$ and $\hat{J}_{Y}$ are $X$ - and $Y$-component spin operators, respectively): Let $\mathcal{H}_{X Y}^{d}$ be the subspace of such vectors for the qudit (base $d$ ) system. For a qutrit, any vector $|\phi\rangle \in \mathcal{H}_{X Y}^{3}$ can be written in the form $|\phi\rangle=\frac{e^{i \alpha}}{2}\left(e^{i \beta}, \sqrt{2}, e^{-i \beta}\right)^{T}$, where $\alpha$ and $\beta$ are real numbers. A convenient choice is the initial state $\left|\psi_{(1)}^{0}\right\rangle=(1, \sqrt{2}, 1)^{T} / 2 \in \mathcal{H}_{X Y}^{3}$, which is the eigenstate of the spin operator $\hat{J}_{X}$. For comparison, in the standard Fourier-based procedure (see Ref. [9] and Appendix D) as optimized for the measurement of a discretely distributed field [i.e., $\omega$ can only take $M$ values $\left\{\omega_{\min }, \omega_{\min }+\Delta \omega, \omega_{\min }+\right.$ $\left.2 \Delta \omega, \ldots, \omega_{\max }\right\}$ with $\left.\Delta \omega=\left(\omega_{\max }-\omega_{\min }\right) / M\right]$ the qutrit is initially prepared in the balanced state $[3](1,1,1)^{T} / \sqrt{3} \notin$ $\mathcal{H}_{X Y}^{3}$. Physically, the larger spin component perpendicular to the field $\left(\left\langle J_{X Y}\right\rangle=1\right.$ in the optimal case versus $2 \sqrt{2} / 3 \approx 0.94$ in the case of the balanced state) ensures a better sensitivity; note that in the qubit case, the balanced initial state $(1,1)^{T} / \sqrt{2}$ already has the largest perpendicular component and thus cannot be further optimized. Turning to the readout gate, our analysis shows that for any $t$, the optimum is always achieved with the Fourier-transform gate

$$
\hat{F}_{3}=\frac{1}{\sqrt{3}}\left(\begin{array}{ccc}
1 & 1 & 1 \\
1 & e^{4 \pi i / 3} & e^{2 \pi i / 3} \\
1 & e^{2 \pi i / 3} & e^{4 \pi i / 3}
\end{array}\right) ;
$$

we remind the reader that for the discretely distributed field the Fourier-transform readout operation is also optimal [3]. 


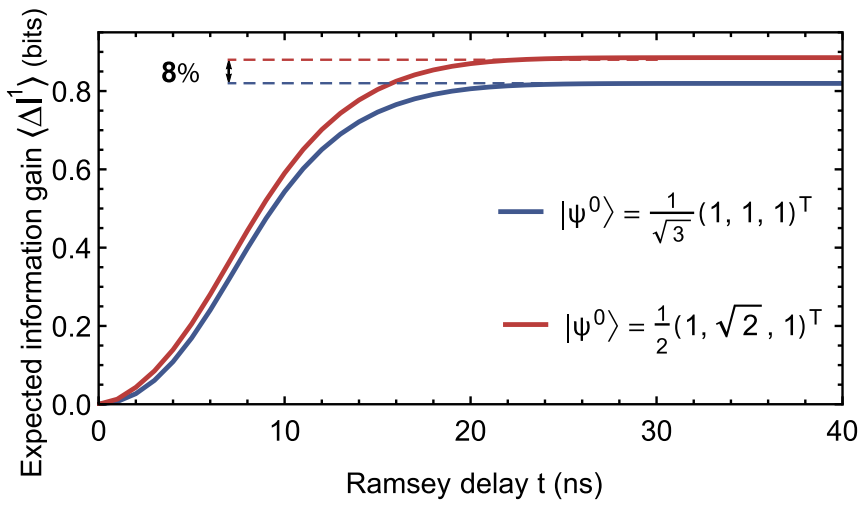

FIG. 1. Information gain for different initial states. Expected information gain during the first measurement step assuming a continuously distributed magnetic field: Blue and red curves correspond to the situations where the qutrit is initially prepared in the balanced state (as in the standard Fourier procedure) and in the state $(1, \sqrt{2}, 1)^{T} / 2 \in \mathcal{H}_{X Y}^{3}$, respectively. The initial field distribution is $\mathcal{P}^{0}(\omega \mid \varnothing)=N\left(0, \sigma^{2}\right)$ with $\sigma=2 \pi /(90 \mathrm{~ns})$. The saturation time is defined by the initial distribution of the magnetic field, $T_{s} \sim 1 / \sigma$; the plateau levels of the blue and red curves are $(5 / 3-\ln 3) / \ln 2 \approx 0.82$ bits and $2\left[(\ln 2)^{-1}-1\right] \approx 0.88$ bits, respectively.

The difference between the standard Fourier-based procedure and the described new optimal procedure [with the initial qutrit state $\left.\left|\psi^{0}\right\rangle=(1, \sqrt{2}, 1)^{T} / 2 \in \mathcal{H}_{X Y}^{3}\right]$ in the continuous situation is illustrated in Fig. 1, where we plot the expected information gain $\left\langle\Delta I^{1}\left(\tilde{\xi}_{1}, t, \mathbf{s}\right)\right\rangle$ in the first step as a function of the Ramsey delay time $t$ with $\mathbf{s}$ corresponding to different initial states and the standard Fourier-transform readout operation. In both cases the information gain saturates at the time $T_{s} \sim 1 / \delta \omega_{0}$, which is defined by the initial field uncertainty $\delta \omega_{0}=\sigma$; a small $\delta \omega_{0}$ increases $T_{s}$, what makes it increasingly harder to further improve the precision, particularly in the presence of decoherence. For the described optimal procedure, the curve reaches a plateau that is higher by $8 \%$, with an information gain of $2\left[(\ln 2)^{-1}-1\right] \approx 0.88$ bits against $(5 / 3-\ln 3) / \ln 2 \approx 0.82$ bits for the balanced state. Hence, when changing from a discrete to a continuous field distribution, the metrological algorithm profits from a refinement in the choice of initial states. We note that any initial state from $\mathcal{H}_{X Y}^{3}$ yields the same (high) plateau level but with the information gain for $t<T_{s}$ depending on the specific choice of $\left|\psi_{(1)}^{0}\right\rangle \in \mathcal{H}_{X Y}^{3}$; as these differences are small, we do not pursue them further here. In the second and further subsequent steps, both scenarios maintain the feature of saturation in the information gain per step, although some interesting new features appear; see Appendix $G$ for a detailed analysis of further optimization steps.

Ordinarily, it is the quantum Fisher information (QFI) estimate which is used as a performance metric. Here, we rather use the information gain as a performance metric, which due to the postselection nature of our sensing protocol appears more appropriate. As described above, the probability distribution of a magnetic field is updated according to the measurement conducted over different quantum states on each step of the algorithm. While the commonly used Cramér-Rao bound, which is based on QFI, gives the asymptotic potential accuracy that can be achieved with a fixed quantum state, our protocol exploits a different approach and, therefore, cannot be analyzed in the context of Fisher information in an evident way.

\section{LINEAR ASCENDING METROLOGICAL ALGORITHM}

Expanding further on the above findings, we develop a novel quantum-metrological procedure exploiting phase coherence which ensures a near-Heisenberg limit scaling even in the presence of dephasing processes when the Kitaev and standard Fourier protocols (see the descriptions in Appendixes $\mathrm{E}$ and $\mathrm{D})$ become ineffective.

The precision of a metrological algorithm is bounded by the maximum possible number of iterations. In the case of the standard Fourier and Kitaev procedures, the limitations are imposed by the coherence time $T_{c}$ and the characteristic duration of the control pulse $T_{p}$ (as described above, the evolution of the transmon qudit is determined by the applied $\mathrm{rf}$ pulses, which cannot be made arbitrarily short and are defined by the hardware): These times bound the Ramsey delay on the longest and shortest time steps of the procedure, respectively. Since in both procedures the delay time of each consequent step changes exponentially (meaning that at each consequent step the delay time is either increased or decreased by factor $d$ ), the number of steps which can be realized in practice is small [8]. One could then think of performing multiple PER steps with the same delay time, as recent state-of-the-art techniques enable rather rapid transmon measurement and reinitialization procedures [26,27]. However, such a routine is essentially classical - hereinafter, this routine will be referred to as the classical procedure-and its precision is therefore restricted by the shot noise limit [6]. To overcome these problems, we propose a novel Linear Ascending Metrological Algorithm, or LAMA, for qudit sensors that combines both classical productivity and quantum scalability and, furthermore, surpasses the efficiency of the Fourier and Kitaev algorithms in common realistic scenarios. The $i$ th step of the LAMA includes the following PER sequence:

$\mathbf{P}$ The qudit is prepared in an initial state $\left|\psi^{0}\right\rangle$ within $\mathcal{H}_{X Y}^{d}$ (the same in every step). In the qutrit case, $\left|\psi^{0}\right\rangle=$ $\frac{e^{i \alpha}}{2}\left(e^{i \beta}|0\rangle+\sqrt{2}|1\rangle+e^{-i \beta}|2\rangle\right)$, where $\alpha$ and $\beta$ are real numbers.

E The qudit is exposed to the magnetic field during the predefined (rather than optimized) time interval $t_{i}^{L}=t_{1}^{L}+$ $(i-1) \Delta t$, with $\Delta t$ chosen of order $\sim 1 / \delta \omega_{0}$, where $\delta \omega_{0}$ is the initial field uncertainty and $t_{1}^{L}=\max \left(T_{p}, T_{S}\right)$.

$\mathbf{R}$ The qudit is subjected to the Fourier transform $\hat{F}_{d}$,

$$
\hat{F}_{d}|n\rangle=\frac{1}{\sqrt{d}} \sum_{k=0}^{d-1} e^{-2 \pi i n k / d}|k\rangle,
$$

and a subsequent single-shot projective measurement of its state in the computational basis. The probability distribution of the magnetic field is updated in accordance with Bayes's formula.

A great simplification of the LAMA is the use of a fixed parameter set $\mathbf{s}_{i}=\mathbf{s}$ for preparation and readout that no longer requires further optimization after each step. The following discussion of the algorithm concerns again the case of a 


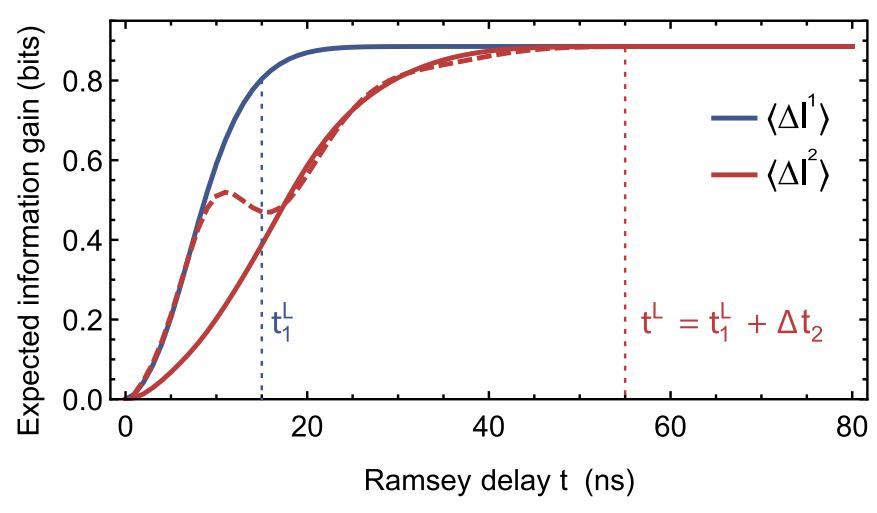

FIG. 2. First two steps of LAMA. Expected information gain $\left\langle\Delta I^{1,2}\right\rangle$ in the first (blue) and second (red) steps of the LAMA as a function of Ramsey delay time $t$. The initial qutrit state is $\left|\psi^{0}\right\rangle=$ $(1, \sqrt{2}, 1)^{T} / 2 \in \mathcal{H}_{X Y}^{3}$, and the initial field distribution is a Gaussian $\mathcal{P}^{0}(\omega \mid \varnothing)=N\left(0, \sigma^{2}\right)$ with $\sigma=2 \pi /(90 \mathrm{~ns}) . t_{1}^{L}$ and $t_{2}^{L}$ are the selected delay times on the first and second steps, respectively. The gain in the second step depends on the outcome of the first step; see dashed red curve when 0 is measured and solid red curve for outcomes 1 or 2 .

continuously distributed field and refers to the example of a qutrit with the optimized initial state $\left|\psi^{0}\right\rangle=(1, \sqrt{2}, 1)^{T} / 2 \in$ $\mathcal{H}_{X Y}^{3}(\alpha=\beta=0)$, different from the balanced one. While the qubit implementation of the new algorithm cannot make use of a further optimization of the initial state, the linear increase in the delay time $t_{i}$ characteristic of the LAMA is still advantageous.

To see how the algorithm works, we first examine the case of an ideal hardware in the absence of decoherence, $T_{c} \rightarrow \infty$. Figure 2 compares the information gain in the second step (red; solid and dashed curves correspond to different outcomes of the first step) with the result obtained in the first step (blue) of the algorithm as a function of the delay time $t$. In order to obtain the maximal information in the first step within the shortest time interval, we choose the delay time at the onset of the saturation plateau, $t_{1}^{L}=T_{s}$. The saturation time $T_{s} \sim 1 / \sigma$ is determined by the expected search range of magnetic fields $\left[\omega_{\min }, \omega_{\max }\right]$ that represents the dynamic range of the sensing device. In Fig. 2, as well as in the following numerical analysis, we set $\sigma=2 \pi /(90 \mathrm{~ns})$, which ensures the maximum achievable dynamic range at reasonable saturation time of the expected information gain. This choice of $\sigma$ gives $T_{s} \approx 15 \mathrm{~ns}$, which corresponds to the fastest possible manipulations of transmons: It becomes unfeasible to control superconducting devices using $T_{p}<10^{-8} \mathrm{~s}$ rf pulses; thus dynamics at shorter times is not meaningful for metrological purposes. In the second step, the expected information gain again saturates at large delay $t_{2}^{L}$ but is reduced at small times $\sim t_{1}^{L}$. This reduction expresses the fact that we have already obtained some information associated with the previous delay time $t_{1}^{L}$; nevertheless, due to the probabilistic nature of the quantum procedure, the prospective information gain remains nonzero. Depending on the outcome of the first step, the gain in the second step may or may not exhibit an additional peak at a delay time below $t_{1}^{L}$; see the dashed curve. The appearance of such a new peak in the information gain in step 2 depends on the relation between the previous outcome $\xi_{1}$ and the chosen initial state for step 2 within the $X Y$ plane. The nature of such dependence is the following: We update the field probability distribution based on the measurement outcome, reducing the distribution's variance at each step. The mean of the distribution indicates the most probable magnetic field value, and it drifts to the true value during the sensing procedure. For the qutrit case, while the $\xi_{1}=0$ outcome does not change the mean of the distribution, $\xi_{1}=1$ and 2 outcomes shift the mean on the right and on the left, respectively. Besides this feature, with a proper choice of $\mathbf{s}_{2}$ the additional peak in the information gain appears at $3 t_{1}^{L}$ ( $d t_{1}^{L}$ for a qudit), where the next step of the Kitaev procedure should be conducted; the correspondence of the appearing peak with the standard Fourier-based procedure is discussed in Appendix G. Instead of attempting to extract this peak information gain by choosing the new initial state parameters $\mathbf{s}_{2}$ in accordance with the previous outcome, we prepare the qutrit in the same initial state (with parameters $\mathbf{s}_{1}$ ) and adopt a time step $t_{2}^{L}=t_{1}^{L}+\Delta t$ with $\Delta t=C / \delta \omega_{0}, \delta \omega_{0}=\sigma$. Evaluation of the optimal prefactor $C$ for each step requires substantial computational time; therefore we will not concentrate on this optimization. Instead, we numerically find the optimal $C \approx \pi$ and fix it for the whole metrological procedure. As we will show further, this choice of a constant time step already ensures performance which, in the presence of decoherence, beats both the classical procedure and the Fourier and Kitaev quantum procedures. This choice of $\Delta t$ and $t_{2}^{L}$ again enables us to exploit the information gain near the plateau of step 2 and learn nearly 0.88 bits of information. The subsequent steps follow the same route: Regardless of the previous outcomes, the qutrit is always prepared in the same state parametrized by $\mathbf{s}_{1}$, while the linear increase in the delay time allows us to operate away from the emerging drops in the information gain, ensuring the advantage of the LAMA over the classical procedure. This straightforward algorithm then provides a great simplification as compared with the other algorithms involving Bayesian learning.

Shown in Fig. 3(a) are the numerically simulated probability distributions for the magnetic field for a six-step procedure; in turn, Fig. 3(b) displays a series of expected information gains [see Eq. (4)] before the next measurement. As the algorithm proceeds, the extracted information per step decreases below the saturation limit of 0.88 bits with the plateau level first shifting farther out to longer delay times and then decreasing over the entire time interval. Nevertheless, as shown below, the practical realization of the LAMA can be quite beneficial in terms of the total information accumulation and scalability. More three-dimensional (3D) plots such as Fig. 3 for different sets of outcomes are presented in Appendix $\mathrm{H}$.

Next, we compare the LAMA with the existing metrological procedures for the experimental situation where the initial field distribution is continuous and the qutrit is subject to decoherence, i.e., $T_{c}$ is finite; details on how our model accounts for dephasing and relaxation are given in Appendix C. For simplicity, we assume that the minimal pulse duration $T_{p}$ is much smaller than the saturation time $T_{s}$ in the first step, which limits the delay time $t_{1}^{L}=\max \left(T_{p}, T_{s}\right)$. We base our comparison on a numerical simulation constituting a series of $10^{3}$ separate computational experiments. In each 

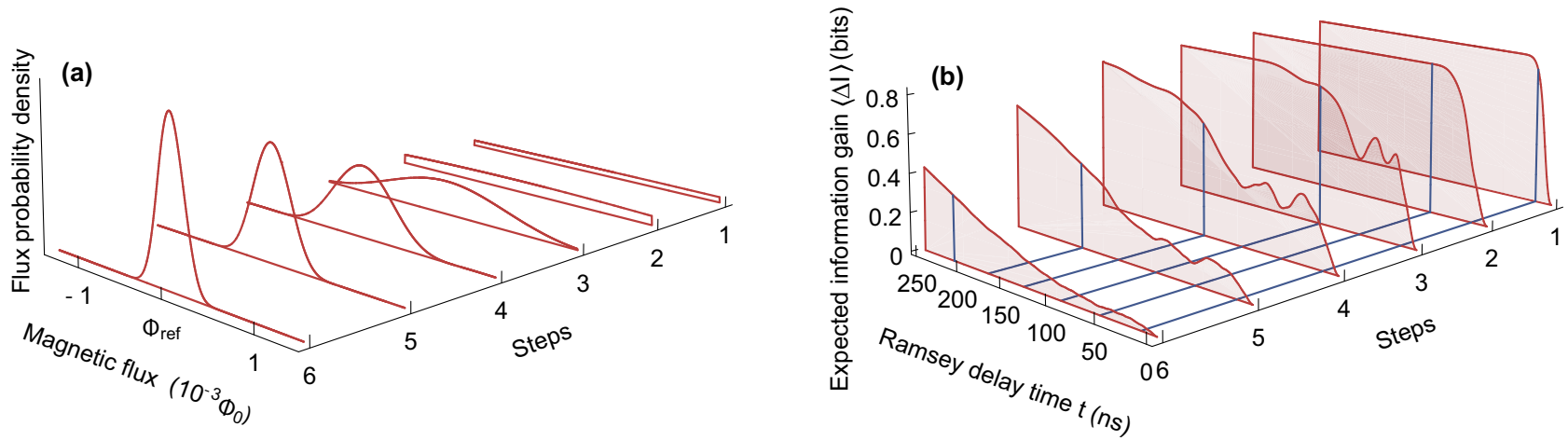

FIG. 3. LAMA operation. (a) Field distributions and (b) expected information gains (before subsequent measurement) at six different steps of the new algorithm as obtained through numerical simulation without dephasing. The initial distribution function is $\mathcal{P}^{0}(\omega \mid \varnothing)=N\left(0, \sigma^{2}\right)$ with $\sigma=2 \pi /(90 \mathrm{~ns})$; the outcomes of the steps are chosen $\left\{\xi_{1}, \xi_{2}, \ldots, \xi_{6}\right\}=\{0,0, \ldots, 0\}$. Given our choice of small delay times, the field distribution in all of the steps is given by an ever-narrowing single peak. We translate the value of reduced magnetic field $\omega$ into the magnetic flux $\Phi=\omega_{01}\left|d \Phi / d \omega_{01}\right|\left(\omega_{01}\right.$ is the transition frequency) by putting [8] $\mu=10^{5} \mu_{0}$, where $\mu_{0}$ is the Bohr magneton. In (b), the delay times at each step are marked by the vertical lines.

experiment, we numerically perform 50 sequential steps of the LAMA in accordance with the above scheme, keeping the parameters $T_{c}, T_{s}, t_{1}^{L}$, and $\Delta t$ the same for every experiment. The outcomes of the simulated experiments are generated randomly: In the $n$th step, the computer samples the outcome $\xi_{n}$ from the probability distribution $P\left(\xi_{n} \mid t_{n}\right)=$ $\int P\left(\xi_{n} \mid \omega^{\prime}, t_{n}\right) \mathcal{P}^{n-1}\left(\omega^{\prime} \mid\left\{\xi_{i}, t_{i}\right\}_{i=1}^{n-1}\right) d \omega^{\prime}$. To determine the efficiency of the procedure, we compose the results of all computational experiments; that is, we plot the information gain averaged over the series of individual experiments as a function of the total phase accumulation time. We compare the LAMA with other algorithms through simulating the operations of the latter in an analogous manner, although the number of steps in the individual experiments may change as required by the different algorithms.

The results of the simulations are summarized in Fig. 4(a), where we show the total information gain as a function of the phase accumulation time $t_{\phi}=\sum_{i} t_{i}$ (the sequence $t_{i}$ denotes all Ramsey delay times). The plot demonstrates that for a typical device and almost any total phase accumulation time $t_{\phi}$, the LAMA allows for a larger information gain, thus providing a higher precision, as compared with the more intricate standard Fourier and Kitaev protocols. This is particularly evident for large accumulation times $t_{\phi} \gtrsim T_{c}$ when the LAMA surpasses other algorithms by $\gtrsim 30 \%$, whereas the standard Fourier and Kitaev procedures provide no significant advantage over the trivial classical procedure.

Finally, we analyze the scaling behavior of the new LAMA. The efficiency of a metrological procedure is reflected in the dependence of the field uncertainty on the total phase accumulation time [8], $\delta \omega\left(t_{\phi}\right) \propto t_{\phi}^{-\alpha}$. A proper quantum algorithm should exceed the shot noise limit of $\alpha=0.5$, ideally reaching the Heisenberg limit $\alpha=1$. Since for any accumulation time $t_{\phi}$ the total information gain is given by $I \simeq-\ln \left[\delta \omega\left(t_{\phi}\right)\right]+$ $\ln [\delta \omega(0)]$ [see Eq. (3)], the scaling can be analyzed in a plot of $I$ as function of $\ln t_{\phi}$. The linear-log plot in Fig. 4(b) displays the scaling behavior of the classical [in black, same curve for any (classical) delay time $t^{C} \ll T_{c}$; if $t^{C} \gtrsim T_{c}$, the procedure operates below the shot noise limit] and standard Fourier and Kitaev procedures (in green, three curves for $T_{c}=\{5,10,30\}$ $\mu \mathrm{s}$ ), as well as the new LAMA (in red, $T_{c}=5 \mu \mathrm{s}$ ) for the continuous field with decoherence included. The curves corresponding to the standard Fourier and Kitaev procedures (green) are indistinguishable. The scaling parameter $\alpha$ can be obtained from the slope of a tangent to a curve at any particular point. As one expects, for any $T_{c}$ larger than the delay time of a step, the classical procedure complies with the shot noise limit, $\alpha=0.5$. We also see that although for $t_{\phi} \ll T_{c}$ the standard Fourier and Kitaev procedures approach the Heisenberg limit, for $t_{\phi} \sim T_{c}$, the value of $\alpha$ drops almost to zero. In turn, the new LAMA, though behaving classically for small $t_{\phi}$, becomes much more efficient than the Fourier and Kitaev procedures for $t_{\phi} \sim T_{c}$, with $\alpha$ reaching its maximum $\simeq 1$ in an intermediate region at $t_{\phi} \simeq 1.1 T_{c}$, at which point the delay time per step $t_{i}^{L} \simeq T_{c} / 7$. One can see that a finite coherence time $T_{c}$ does not posit any notable limitation on the LAMA's efficiency until the delay time $t_{i}^{L}$ becomes comparable with $T_{c}$; at large accumulation $t_{\phi} \sim 10 T_{c}$ the algorithm still operates close to the shot noise limit with $\alpha \gtrsim 0.5$. Importantly, we see that even when implemented on a mediocre transmon qutrit device with $T_{c}=5 \mu \mathrm{s}$, the new LAMA is capable of outperforming other algorithms realized with a cutting-edge device with $T_{c}=30 \mu \mathrm{s}$ [28].

\section{CONCLUSION}

In summary, we have proposed a simple and robust sequential quantum-metrological algorithm for magnetometry, the LAMA, which is characterized by two important features: (i) Each measurement step involves a linear increase in the Ramsey delay time, and (ii) the qudit is always initialized in the same state of maximal spin perpendicular to the field. The prescription of these measurement parameters drastically reduces the complexity of the algorithm as compared with algorithms requiring learning. The linear increase in the Ramsey delay time guarantees an improved performance as compared with the classical algorithm, where the repeated measurement at the same Ramsey time produces a steadily reduced information gain with each additional step. We have compared our algorithm with quantum Fourier and Kitaev algorithms 

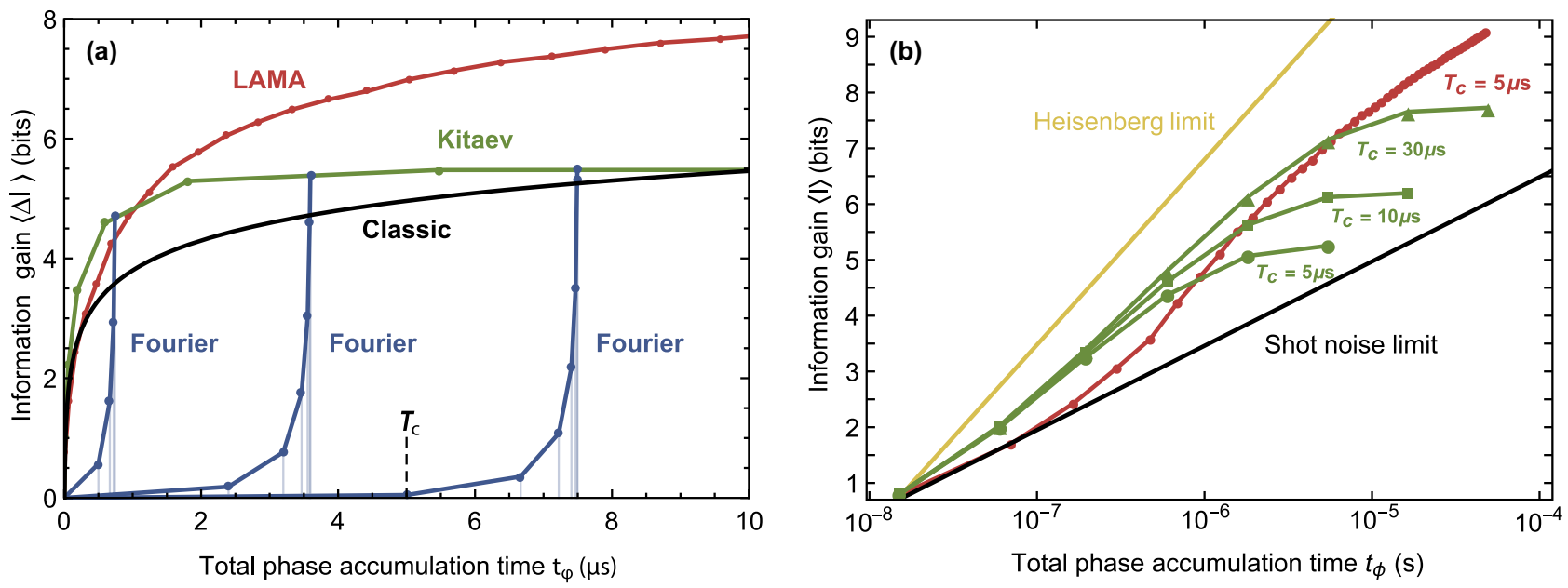

FIG. 4. Comparison of the algorithms for a transmon qutrit. (a) Linear and (b) linear-log plots of the information gain defined in Eq. (4) in the classical (black), Kitaev (green), and standard Fourier (blue) procedures as functions of the total phase accumulation time; the red curve provides the result for the new LAMA. The symbols mark the individual steps of the four procedures. All curves are averaged over a series of $10^{3}$ numerical experiments (for details of the simulation, see the main text). We have chosen $T_{s}=15 \mathrm{~ns}, \mathcal{P}^{0}(\omega \mid \varnothing)=N\left(0, \sigma^{2}\right)$ with $\sigma=2 \pi /(90 \mathrm{~ns})$ and $T_{p}<T_{s}$. The initial delay time in the classical, $t_{1}^{C}$, and Kitaev, $t_{1}^{K}$, procedures and in the LAMA, $t_{1}^{L}$, is $15 \mathrm{~ns}$; in the case of the LAMA, the time increase per step is $\Delta t=40 \mathrm{~ns}$. In (a) the standard Fourier procedure is represented by three curves corresponding to different initial delay times: $t_{1}^{F}=0.5,2.4$, and $5 \mu \mathrm{s}$. In the case of the Fourier procedure, a larger $t_{1}^{F}$ allows us to perform more steps of the algorithm since on each step we are decreasing time from $t_{1}^{F}$ to $T_{s}$. However, due to the decoherence, it also results in an insufficient information gain in the first steps; $t_{1}^{F}$ approaches $T_{c}$. In the case of the Kitaev procedure, decoherence affects last steps since they are performed at larger delay times. The final gain in the Fourier case corresponds to the gain obtained with the Kitaev procedure at the same total phase accumulation time. Note that the highest points of the blue curves collapse onto the green curve. This is not a surprise since the Fourier and Kitaev procedures are expected to provide the same performance; for that reason we do not plot curves for the Fourier procedure in (b). All curves in (a) are obtained for the same coherence time $T_{c}=5 \mu \mathrm{s}$, whereas in (b), we show three green curves corresponding to the Kitaev procedure for three different values of $T_{c}, 5 \mu \mathrm{s}$ (circles), $10 \mu \mathrm{s}$ (squares), and $30 \mu \mathrm{s}$ (triangles); the red and black curves referring to the LAMA and to the classical procedure are obtained for $T_{c}=5 \mu \mathrm{s}$. The yellow straight line marks the Heisenberg quantum limit with scaling parameter $\alpha=1$.

and demonstrated that the LAMA provides a markedly better performance in the realistic situation where the qudit is subject to decoherence and the measured field is distributed continuously. As the decoherence limits the delay time range, the LAMA should enable us to utilize the resource of quantum coherence more effectively: In comparison with other algorithms, the LAMA better spans the full available range of times and thus allows for more iterations and better scaling. The presented results have far-reaching implications going beyond the context in which they were derived. We anticipate that our findings will accelerate progress towards reliable quantum magnetic sensors and find use in other applications.

\section{ACKNOWLEDGMENTS}

We are grateful to S. N. Filippov, G. G. Amosov, and G. S. Paraoanu for valuable discussions. This work was supported by the Government of the Russian Federation (Agreement No. 05.Y09.21.0018), Grants No. 17-02-00396A, No. 1802-00642A, and No. 19-32-80005 (N.S.K. and M.R.P.), the Foundation for the Advancement of Theoretical Physics and Mathematics "BASIS," the Ministry of Education and Science of the Russian Federation, the Swiss National Foundation via the National Centre of Competence in Research in Quantum Science and Technology (NCCR QSIT) (G.B.). The work of V.M.V. was supported by Terra Quantum AG.
M.R.P. and N.S.K. contributed equally to this work; N.S.K. proposed the algorithm; M.R.P., N.S.K., and V.V.Z. performed calculations and analyzed the numerical data; N.S.K., M.R.P., V.M.V., and G.B. wrote the manuscript with input from all authors; G.B.L., G.B., and V.M.V. supervised the project; all authors discussed the results and contributed to the work.

\section{APPENDIX A: TRANSMON PHASE ACCUMULATION DYNAMICS}

Here, we discuss the working principles of the transmon device in the context of magnetic field sensing; an extended discussion on the subject can be found in Ref. [9]. Let us examine the evolution of the transmon device operating in a qudit mode (having effectively $d$ energy levels) in the presence of the magnetic field. The transmon's transition energies can be described to leading order by the expression

$$
\begin{aligned}
E_{n, n+1}(\omega) & =E_{n+1}(\omega)-E_{n}(\omega) \\
& =\sqrt{8 E_{J}(\omega) E_{c}}-E_{c}(n+1),
\end{aligned}
$$

where $E_{n}$ is the energy of the $n$th level, $E_{J}(\omega)$ is the Josephson energy sensitive to the field $\omega$, and $E_{c}$ is the charging energy. Note that the dependence of the different transition energies on the magnetic field is identical, as it is determined solely by $E_{J}(\omega)$. As a result, the first-order correction to the energy separation $E_{0, n}=E_{n}-E_{0}$ in the presence of the magnetic 
field is given by

$$
\partial_{\omega} E_{0, n} \omega=n \partial_{\omega} E_{0,1} \omega
$$

for any $n \in\{0, \ldots, d-1\}$. Thus the phase accumulation due to the field is linear in $n$, and we obtain the following expression for the effective evolution operator in the rotating frame approximation:

$$
\hat{U}^{e}=e^{-i\left(\operatorname{diag}\left[0, \partial_{\omega} E_{0,1} \omega t, 2 \partial_{\omega} E_{0,1} \omega t, \ldots,(d-1) \partial_{\omega} E_{0,1} \omega t\right]\right)} .
$$

For simplicity, in what follows we put $\partial_{\omega} E_{0,1}=1$. The above linear phase accumulation dynamics is crucial for the implementation of all algorithms discussed in this paper. At the same time, the LAMA can be realized on any other multilevel system beyond the transmon, provided that the level separations scale equally in the magnetic field.

\section{APPENDIX B: OPTIMIZATION METHOD}

Here, we elaborate on the general qutrit realization of the PER protocol in the $i$ th step [9] (all matrices are represented in the computational basis $|k\rangle, k=\{0,1,2\})$ :

$\mathbf{P}$ The qutrit is initialized through the application of a two-tone rectangular rf pulse [9] to the ground state $|0\rangle$. The unitary evolution induced by the pulse has the form

$$
\hat{U}_{(i)}^{p}=\exp \left(-i\left[\begin{array}{ccc}
0 & \Delta_{1(i)}^{p} & 0 \\
\Delta_{1(i)}^{p} & 2 \epsilon_{(i)}^{p} & \Delta_{2(i)}^{p} \\
0 & \Delta_{2(i)}^{p} & 0
\end{array}\right]\right)
$$

and results in the state $\left|\psi_{(i)}^{0}\right\rangle=\hat{U}_{(i)}^{p}|0\rangle$. Here, $\Delta_{1,2(i)}^{p}$ are effective transition amplitudes between qubit states, and $\epsilon_{(i)}^{p}$ is related to the pulse frequencies; their optimal values can be found by numerical optimization [9].

E The qutrit interacts with the external magnetic field $H$ during time $t_{i}:\left|\psi_{(i)}^{0}\right\rangle \rightarrow\left|\psi_{(i)}^{t_{i}}\right\rangle$. The field changes the phases inside the state vector: $|k\rangle \rightarrow e^{i k \omega t_{i}}|k\rangle(k=\{0,1,2\})$, where we omitted a common phase factor. In the absence of decoherence, the evolution operator is given by

$$
\hat{U}_{(i)}^{e}=\exp \left(-i\left[\begin{array}{ccc}
0 & 0 & 0 \\
0 & \omega t_{i} & 0 \\
0 & 0 & 2 \omega t_{i}
\end{array}\right]\right) .
$$

$\mathbf{R}$ The qutrit undergoes a readout operation generated again by the pulse

$$
\hat{U}_{(i)}^{r}=\exp \left(-i\left[\begin{array}{ccc}
0 & \Delta_{1(i)}^{r} & 0 \\
\Delta_{1(i)}^{r} & 2 \epsilon_{(i)}^{r} & \Delta_{2(i)}^{r} \\
0 & \Delta_{2(i)}^{r} & 0
\end{array}\right]\right) .
$$

Finally, the information about the field value is extracted through the single-shot projective measurement of $\left|\psi_{(i)}^{f}\right\rangle=$ $\hat{U}_{(i)}^{r}\left|\psi_{(i)}^{t_{i}}\right\rangle$ in the computational basis.

The above PER sequence yields a fairly cumbersome expression for the final state (before the measurement) $\left|\psi_{(i)}^{f}\right\rangle=$ $\hat{U}_{(i)}^{r} \hat{U}_{(i)}^{e} \hat{U}_{(i)}^{p}|0\rangle$, and we refrain from providing it here.

The optimization of the $(n+1)$ st step for a given delay time $t$ attempts to find the preparation and readout parameters $\mathbf{s}_{n+1}=\left(\epsilon_{(n+1)}^{p}, \Delta_{1(n+1)}^{p}, \Delta_{2(n+1)}^{p}, \epsilon_{(n+1)}^{r}, \Delta_{1(n+1)}^{r}, \Delta_{2(n+1)}^{r}\right)$ that produce the maximum expected information gain on that step,

$$
\begin{aligned}
& \left\langle\Delta I^{n+1}\left(\left\{\xi_{i}, t_{i}, \mathbf{s}_{i}\right\}_{i=1}^{n},\left\{\tilde{\xi}_{n+1}, t, \mathbf{s}_{n+1}\right\}\right)\right\rangle \\
& \quad=\max _{\mathbf{s}}\left\langle\Delta I^{n+1}\left(\left\{\xi_{i}, t_{i}, \mathbf{s}_{i}\right\}_{i=1}^{n},\left\{\tilde{\xi}_{n+1}, t, \mathbf{s}\right\}\right)\right\rangle,
\end{aligned}
$$

where the averaging is done in accordance with Eqs. (4) and (5).

To implement and optimize numerically the above procedure, we model the continuous field by an evenly spaced grid with the large number of points $M=10^{5}$; the initial field values are Gaussian weighted, $\mathcal{P}^{0}(\omega \mid \varnothing)=N\left(0, \sigma^{2}\right)$ with $\sigma=$ $2 \pi /(90 \mathrm{~ns})$. We choose $\sigma$ in a way that the saturation time of the expected information gain is $T_{s} \approx 15 \mathrm{~ns}$ - it becomes impracticable to apply gates faster than $10^{-8} \mathrm{~s}$ to transmon devices. In the first step, the optimal choice of $\mathbf{s}_{1}$ for a given $t$ corresponds to the maximum of the averaged information gain $\left\langle\Delta I^{1}\left(\tilde{\xi}_{1}, t, \mathbf{s}\right)\right\rangle$. Since for the numerical calculations the continuous distribution is modeled by a discrete one, the integral with respect to $\omega^{\prime}$ has to be replaced by a sum

$$
\begin{aligned}
\left\langle\Delta I^{1}\left(\tilde{\xi}_{1}, t, \mathbf{s}\right)\right\rangle & \\
= & -\sum_{m=1}^{M} \mathcal{P}^{0}\left(\omega_{m} \mid \varnothing\right) \ln \mathcal{P}^{0}\left(\omega_{m} \mid \varnothing\right) \\
& -\sum_{\tilde{\xi}_{1}=0}^{2} \sum_{m=1}^{M} S^{1}\left(\tilde{\xi}_{1}, t, \mathbf{s}\right) P\left(\tilde{\xi}_{1} \mid \omega_{m}, t, \mathbf{s}\right) \mathcal{P}^{0}\left(\omega_{m} \mid \varnothing\right) .
\end{aligned}
$$

Here, $P\left(\tilde{\xi}_{1} \mid \omega_{m}, t, \mathbf{s}\right)$ is given by Eq. (1); the expression for $S^{1}\left(\tilde{\xi}_{1}, t, \mathbf{s}\right)$ can be obtained from Eqs. (2) and (3),

$$
\begin{aligned}
S^{1}\left(\tilde{\xi}_{1}, t, \mathbf{s}\right)= & -\sum_{m=1}^{M} \frac{\mathcal{P}^{0}\left(\omega_{m} \mid \varnothing\right) P\left(\tilde{\xi}_{1} \mid \omega_{m}, t, \mathbf{s}\right)}{\sum_{m^{\prime}=1}^{M} \mathcal{P}^{0}\left(\omega_{m^{\prime}} \mid \varnothing\right) P\left(\tilde{\xi}_{1} \mid \omega_{m^{\prime}}, t, \mathbf{s}\right)} \\
& \times \ln \frac{\mathcal{P}^{0}\left(\omega_{m} \mid \varnothing\right) P\left(\tilde{\xi}_{1} \mid \omega_{m}, t, \mathbf{s}\right)}{\sum_{m^{\prime}=1}^{M} \mathcal{P}^{0}\left(\omega_{m^{\prime}} \mid \varnothing\right) P\left(\tilde{\xi}_{1} \mid \omega_{m^{\prime}}, t, \mathbf{s}\right)} .
\end{aligned}
$$

\section{APPENDIX C: RELAXATION AND DEPHASING PROCESSES}

We extend our model to account for the decoherence processes appearing in the transmons. When the Ramsey delay time $t_{i}$ becomes comparable to the coherence time $T_{c}$, the state analysis requires solving a kinetic equation, which we choose to be of the Lindblad form [29]

$$
\frac{d \hat{\rho}}{d t}=-i\left[\hat{\rho}, \hat{H}_{\mathrm{int}}\right]+\Gamma_{01} \hat{D}\left[\sigma_{01}\right] \hat{\rho}+\Gamma_{12} \hat{D}\left[\sigma_{12}\right] \hat{\rho},
$$

where $\hat{H}_{\text {int }}$ is the interaction Hamiltonian

$$
\hat{H}_{\text {int }}=\hbar\left(\begin{array}{ccc}
0 & 0 & 0 \\
0 & \omega & 0 \\
0 & 0 & 2 \omega
\end{array}\right),
$$

$\Gamma_{i j}$ are the energy relaxation rates, and the superoperator $\hat{D}$ describes the process of energy relaxation,

$$
\hat{D}\left[\sigma_{i j}\right] \hat{\rho}=\hat{\sigma}_{i j} \hat{\rho} \hat{\sigma}_{i j}^{\dagger}-\frac{1}{2}\left\{\hat{\sigma}_{i j}^{\dagger} \hat{\sigma}_{i j}, \hat{\rho}\right\},
$$

with the Lindblad operators $\hat{\sigma}_{i j}=|i\rangle\langle j|(i, j=\{0,1,2\})$. In addition, we consider fluctuations $\delta H$ of the field due to 
fluctuating electric currents or magnetic impurities, assuming Gaussian noise parametrized by the dephasing rate $\Gamma_{\varphi}$,

$$
\left\langle\delta H(t) \delta H(t)^{\prime}\right\rangle=\left(\frac{d \omega}{d H}\right)^{-2} \Gamma_{\varphi} \delta\left(t-t^{\prime}\right) .
$$

The above extensions are incorporated in our PER procedure in the following manner:

$\mathbf{P}$ In the $i$ th step, the qutrit is put in the initial state defined by the density matrix $\hat{\rho}_{(i)}^{0}=\left|\psi_{(i)}^{0}\right\rangle\left\langle\psi_{(i)}^{0}\right|$.

$\mathbf{E}$ The qutrit interacts with the external magnetic field $H$ with corresponding changes of the phases in the density matrix, which is furthermore affected by the decoherence processes, $\hat{\rho}_{(i)}^{0} \rightarrow \hat{\rho}_{(i)}\left(t_{i}\right)$. The elements $\rho_{(i)}^{p q}\left(t_{i}\right)(p, q \in\{0,1,2\})$ of the resulting density matrix can be expressed through the elements of $\hat{\pi}_{(i)}\left(t_{i}\right)$ describing the qutrit state after the interaction in the absence of decoherence,

$$
\begin{aligned}
\rho_{(i)}^{22}\left(t_{i}\right)= & \pi_{(i)}^{22}\left(t_{i}\right) e^{-\Gamma_{21} t_{i}}, \\
\rho_{(i)}^{11}\left(t_{i}\right)= & \pi_{(i)}^{11}\left(t_{i}\right) e^{-\Gamma_{10} t_{i}} \\
& +\pi_{(i)}^{22}\left(t_{i}\right) \frac{\Gamma_{21}}{\Gamma_{21}-\Gamma_{10}}\left(e^{\left(\Gamma_{21}-\Gamma_{10}\right) t_{i}}-1\right), \\
\rho_{(i)}^{00}\left(t_{i}\right)= & 1-\rho_{(i)}^{11}\left(t_{i}\right)-\rho_{(i)}^{22}\left(t_{i}\right), \\
\rho_{(i)}^{01}\left(t_{i}\right)= & \pi_{(i)}^{01}\left(t_{i}\right) e^{-\frac{\left(\Gamma_{10}+\Gamma_{\varphi}\right) t_{i}}{2}}, \\
\rho_{(i)}^{10}\left(t_{i}\right)= & \pi_{(i)}^{10}\left(t_{i}\right) e^{-\frac{\left(\Gamma_{10}+\Gamma \varphi\right) t_{i}}{2}}, \\
\rho_{(i)}^{02}\left(t_{i}\right)= & \pi_{(i)}^{02}\left(t_{i}\right) e^{-\frac{\left(\Gamma_{21}+4 \Gamma_{\varphi}\right) t_{i}}{2}}, \\
\rho_{(i)}^{20}\left(t_{i}\right)= & \pi_{(i)}^{20}\left(t_{i}\right) e^{-\frac{\left(\Gamma_{21}+4 \Gamma_{\varphi}\right) t_{i}}{2}}, \\
\rho_{(i)}^{12}\left(t_{i}\right)= & \pi_{(i)}^{12}\left(t_{i}\right) e^{-\frac{\left(\Gamma_{21}+\Gamma_{01}+\Gamma_{\varphi}\right) t_{i}}{2}}, \\
\rho_{(i)}^{21}\left(t_{i}\right)= & \pi_{(i)}^{21}\left(t_{i}\right) e^{-\frac{\left(\Gamma_{21}+\Gamma_{01}+\Gamma \varphi\right) t_{i}}{2}} .
\end{aligned}
$$

R The qutrit undergoes the readout operation $\hat{U}_{(i)}^{r}$, and the final state is subjected to the single-shot projective measurement.

As an example, we consider the first step of the standard Fourier procedure in the presence of dephasing. Using the above formulas and Eq. (1), we can write the probability of finding the qutrit in state $\left|\xi_{1}\right\rangle$ in the form

$$
\begin{aligned}
P\left(\xi_{1} \mid \omega, t\right)= & \left|\left\langle\xi_{1}\left|\hat{U}_{(1)}^{r} \hat{\rho}_{(1)}\left(t_{i}\right) \hat{U}_{(1)}^{r \dagger}\right| \xi_{1}\right\rangle\right|^{2} \\
= & \frac{1}{3}+\frac{2}{9} \cos \left(\omega t-\frac{2 \pi}{3} \xi_{1}\right) e^{-\frac{\Gamma_{10}+\Gamma \varphi}{2} t} \\
& +\frac{2}{9} \cos \left(2 \omega t+\frac{2 \pi}{3} \xi_{1}\right) e^{-\frac{\Gamma_{21}+4 \Gamma_{\varphi}}{2} t} \\
& +\frac{2}{9} \cos \left(\omega t-\frac{2 \pi}{3} \xi_{1}\right) e^{-\frac{\Gamma_{10}+\Gamma_{12}+\Gamma_{\varphi}}{2} t} .
\end{aligned}
$$

In our simulations, we assume $\Gamma_{01}=\Gamma_{12} / \sqrt{2}=\Gamma_{\varphi}=\Gamma$ and describe the decoherence rate by the coherence time $T_{c}=$ $1 / \Gamma$.

\section{APPENDIX D: FOURIER-BASED METROLOGICAL ALGORITHM}

The standard Fourier-based algorithm is a sequence of PER steps performed with different delay times. Including learning, the algorithm involves a conditional initial preparation at each step that depends on the previous outcome. The $i$ th step of the base- 3 procedure can be described by the following scheme:

$\mathbf{P}$ The qutrit is prepared in the state $\left|\psi_{(i)}^{0}\right\rangle=\frac{1}{\sqrt{3}}(|0\rangle+$ $\left.e^{i \alpha_{i}}|1\rangle+e^{2 i \alpha_{i}}|2\rangle\right)$, where $\alpha_{i}=-\frac{2 \pi}{3}\left(\frac{\xi_{i-1}}{3^{1}}+\frac{\xi_{i-2}}{3^{2}}+\cdots+\frac{\xi_{1}}{3^{i-1}}\right)$ and $\xi_{j}$ is the outcome of the $j$ th step (and $\alpha_{1}=0$ ).

$\mathbf{E}$ The system is exposed to the magnetic field during time $t_{i}^{F}=t_{1}^{F} / 3^{i-1}$.

$\mathbf{R}$ The qutrit undergoes the Fourier transform given by Eq. (6). The subsequent single-shot projective measurement of the final state in the computational basis provides the new information on the field. The probability distribution is updated in accordance with Bayes's formula.

In order to illustrate the principle of the algorithm, we consider the situation where the measured field can be expressed in a ternary decomposition,

$$
\omega=\omega_{0}\left(\frac{r_{K}}{3^{0}}+\frac{r_{K-1}}{3^{1}}+\cdots+\frac{r_{1}}{3^{K-1}}\right),
$$

where trits $r_{n}, n \in\{1, \ldots, K\}$ can assume values 0,1 , and 2 .

In the first step, the qutrit is prepared in the balanced state $\left|\psi_{(1)}^{0}\right\rangle=\frac{1}{\sqrt{3}}(|0\rangle+|1\rangle+|2\rangle)$ and is exposed to the field for time $t_{1}^{F}=2 \pi \cdot 3^{K-2} / \omega_{0}$. The qutrit thus assumes the state $\left|\psi_{(1)}^{t_{1}}\right\rangle=i \frac{1}{\sqrt{3}}\left(|0\rangle+e^{i \phi_{1}}|1\rangle+e^{2 i \phi_{1}}|2\rangle\right.$ ) (we omit the overall phase factor $\left.e^{-i \omega t_{1}}\right)$, where $\phi_{1}=(2 \pi / 3) r_{1}$ is the fielddependent phase, which can be unambiguously determined through the application of the Fourier transform and a projective measurement of the final state $\hat{F}\left|\psi_{(1)}^{t_{1}}\right\rangle=\left|r_{1}\right\rangle$; the latter constitutes one of the computational basis vectors $|0\rangle,|1\rangle$, or $|2\rangle$. The outcome of the measurement is $\xi_{1}=r_{1}$.

Turning to the second step and accounting for the first outcome, the qutrit is prepared in the modified balanced state $\left|\psi_{(2)}^{0}\right\rangle=\frac{1}{\sqrt{3}}\left(|0\rangle+e^{i \alpha_{2}}|1\rangle+e^{2 i \alpha_{2}}|2\rangle\right)$ with $\alpha_{2}=$ $-\frac{2 \pi r_{1}}{9}$. After an exposure time $t_{2}^{F}=t_{1}^{F} / 3$, the qutrit evolves to the state $\left|\psi_{(1)}^{t_{1}}\right\rangle=\frac{1}{\sqrt{3}}\left(|0\rangle+e^{i\left(\alpha_{2}+\phi_{2}\right)}|1\rangle+e^{2 i\left(\alpha_{2}+\phi_{2}\right)}|2\rangle\right)$, where $\phi_{2}$ is the field-dependent phase and $\alpha_{2}+\phi_{2}=$ $-\frac{2 \pi}{9} r_{1}+\frac{2 \pi}{3}\left(r_{2}+\frac{r_{1}}{3}\right)=\frac{2 \pi}{3} r_{2}$. The digit $r_{2}$ can then be found through proper readout and measurement as in the previous step. Similarly, the subsequent steps provide the further digits.

\section{APPENDIX E: KITAEV ALGORITHM}

While the standard Fourier procedure allows one to progressively learn the ternary value of the field starting from the "smallest" digit $r_{1}$, the Kitaev algorithm works in the reverse manner outputting the leading digit $r_{K}$ first. The $i$ th step of the base- 3 procedure is as follows:

$\mathbf{P}$ The qutrit is prepared in the state $\left|\psi_{(i)}^{0}\right\rangle=\frac{1}{\sqrt{3}}(|0\rangle+$ $|1\rangle+|2\rangle)$.

E The system is exposed to the magnetic field during the time $t_{i}^{K}=t_{1}^{K} \cdot 3^{i-1}$.

$\mathbf{R}$ The qutrit undergoes a Fourier transform $\hat{F}_{3}$ given by Eq. (6). The information about the field value is extracted 
through the single-shot projective measurement of the final state in the computational basis, and the probability distribution is updated in accordance with Bayes's formula.

As distinct from the standard Fourier procedure, with each step the delay time increases, ensuring that the field distribution is always represented by a single peak.

\section{APPENDIX F: OSCILLATORY FEATURES IN THE INFORMATION GAIN}

In this Appendix, we discuss the oscillatory features intrinsic to the expected information gain as a function of the delay time. The plots describing the information gain and presented in the text [e.g., Fig. 3(b)] have been obtained under the assumption that initially, the field is distributed continuously and the distribution function is Gaussian centered at $\omega=0$. Under these conditions, no oscillatory features show up; the latter are not central to the main discussion, but we shall address them now as they may show up in other circumstances. Given the complexity of the underlying mathematical expressions, our numerical analysis has a rather qualitative character. The analysis concerns the base- 3 standard Fourier procedure (with no dephasing in the system), but the results are also relevant to the other metrological algorithms proposed in the main text.

In general, there are three types of oscillations showing up in the information gain related to the edges, the center position, and the discretization scale of the distribution function. We shall study each of them separately by evaluating the information gain as a function of delay time in the first step of the standard Fourier procedure under different conditions.

Edges. We consider the situation where the field is distributed continuously over the interval $[-\Omega / 2,+\Omega / 2]$. Figure 5(a) displays the information gain for a uniform distribution, with a saturation plateau modulated by oscillations with period $T_{\text {edge }} \propto 1 / \Omega$. Such oscillations are imposed by the abrupt edges of the distribution function and disappear for the case of a Gaussian distribution with smooth tails.

Center position. Another type of oscillation originates from a nonvanishing field average. Figure 5(b) depicts the information gain for a continuous field with a Gaussian distribution centered at different positions $\omega_{\text {center }} \neq 0$ and at $\omega_{\text {center }}=0$. Again, oscillations with a period $T_{\text {center }} \propto 1 /\left|\omega_{\text {center }}\right|$ show up, this time in the rising part of the gain function.

Discreteness. The third type of oscillation appears for the case of a discretely distributed field. In this paper, the continuous case is modeled with a fine-grained discrete distribution built from outcomes with a large number of measurable values. Numerically, the continuous distribution on the interval $\left[\omega_{\min }, \omega_{\max }\right]$ implies that the field can assume $M \sim 10^{5}$ possible values $\left\{\omega_{\min }, \omega_{\min }+\Delta \omega, \omega_{\min }+2 \Delta \omega, \ldots, \omega_{\max }\right\}$ with $\Delta \omega=\left(\omega_{\max }-\omega_{\min }\right) / M$. As revealed in Fig. 5(c), a nonzero spacing $\Delta \omega$ gives rise to a periodic pattern with period $T_{\Delta} \propto$ $1 / \Delta \omega$; the depicted plots are obtained for the case of a Gaussian distribution on the interval $[-\Omega / 2,+\Omega / 2]$ with different values of $M$.

The oscillations appearing in Figs. 7(b), 7(d), and 7(f) presented below are mainly of the second type, as after the first step the center of the distribution shifts away from zero. In
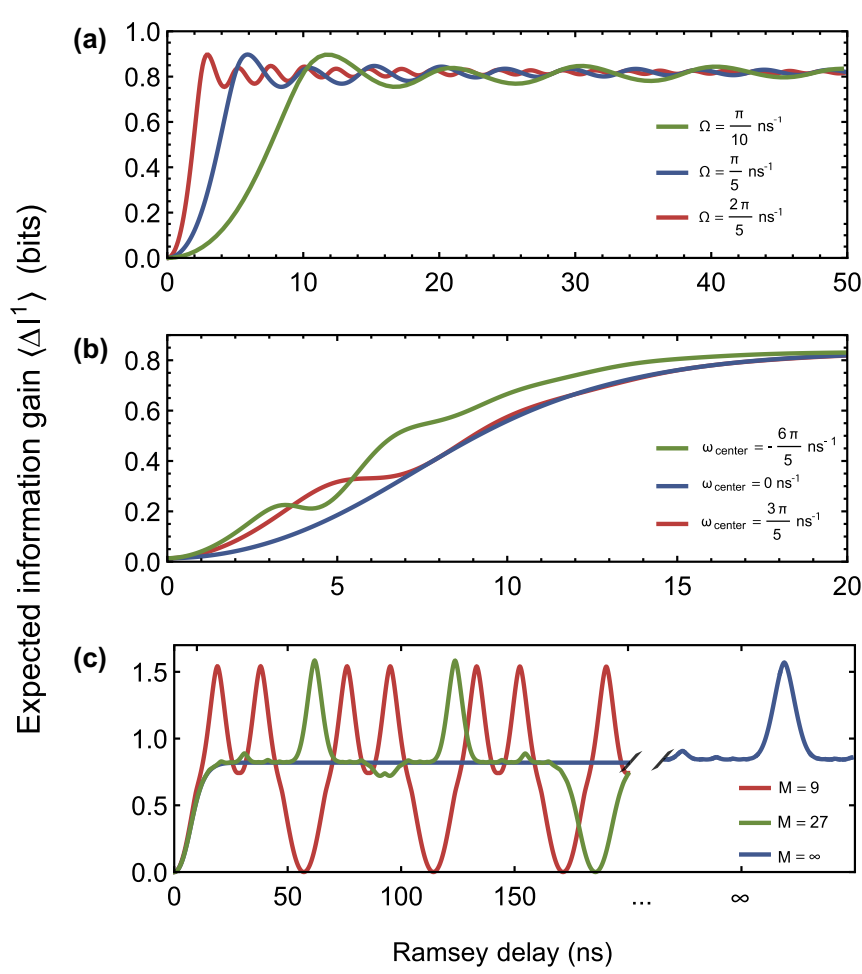

FIG. 5. Oscillations in the information gain. Expected information gain during the first step of the standard Fourier procedure as a function of the delay time evaluated under different conditions. The detailed behavior of the information gain function is determined by the initial field distribution. (a) Information gain for a continuous uniformly distributed initial field with zero mean and continuous sampling for three different sharp intervals of width $\Omega$. Oscillations with period $T_{\text {edge }} \propto \Omega$ appear due to the sharp edges of the uniform distribution. (b) Information gain for a continuous Gaussian initial distribution with $\sigma=2 \pi /(90 \mathrm{~ns})$ and continuous sampling for three different mean values $\omega_{\text {center }}$. The period of the observed oscillations is given by $T_{\text {center }} \propto 1 /\left|\omega_{\text {center }}\right|$. (c) Information gain for a Gaussian initial distribution with $\sigma=2 \pi /(90 \mathrm{~ns})$ and zero mean and different sampling rates. The oscillatory pattern has a period $T_{\Delta} \propto 1 / \Delta \omega$.

addition, oscillations of the third type play an important role. When the field values are discrete with spacing $\Delta \omega>0$, the standard Fourier procedure generates peaks with the largest ones corresponding to the maximum possible information gain of 1 trit $=\log _{2}(3)$ bits. This reaffirms that for the discrete case, the standard Fourier procedure is indeed optimal, given an appropriately chosen delay time. However, this is no longer the case when the field distribution is continuous and $\Delta \omega=0$, since the oscillatory peak would correspond to an infinite delay time. Nevertheless, as we will see in Appendix $\mathrm{G}$, this type of oscillation still manifests itself in the second and subsequent steps of the procedure.

\section{APPENDIX G: STANDARD FOURIER PROCEDURE IN THE CONTINUOUS CASE}

We now consider in more detail the second and further steps of the base-3 standard Fourier procedure for the case of a continuous field distribution when the dephasing is absent. With the delay time in the first step chosen to be relatively 
(a)

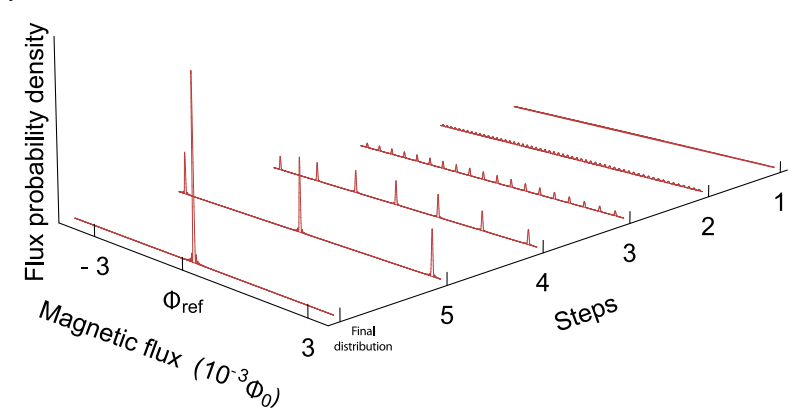

(b)

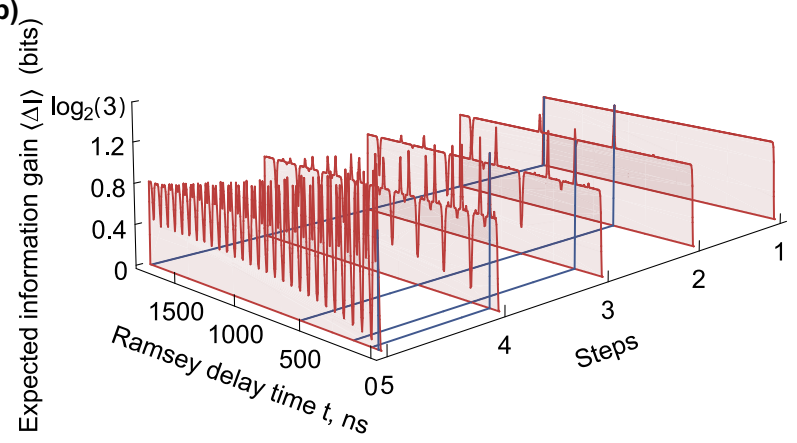

(c) $\stackrel{0}{\stackrel{n}{ \pm}}$

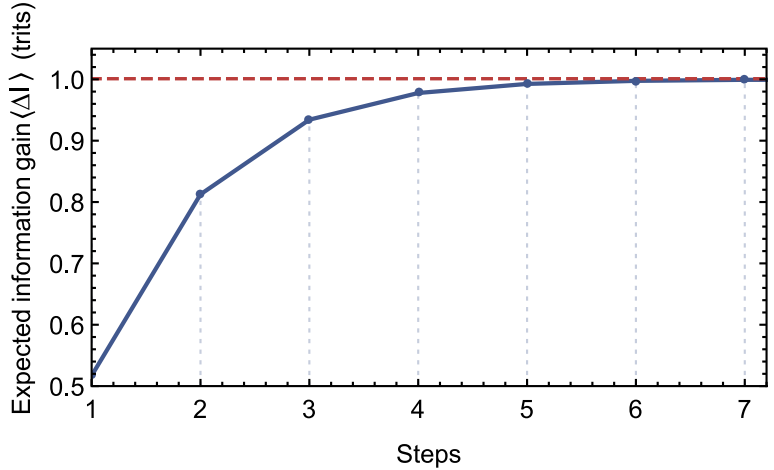

(d) $\frac{\widehat{D}}{\stackrel{0}{0}}$

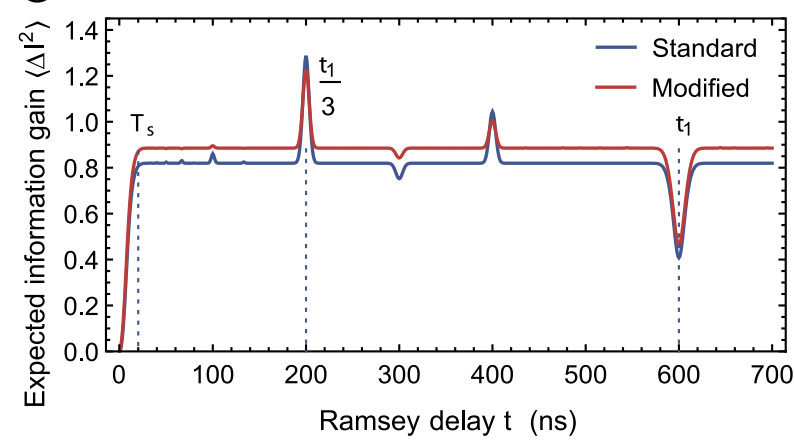

FIG. 6. Standard Fourier algorithm. (a) Field distributions and (b) expected information gains at six different steps of the standard Fourier procedure as obtained through numerical modeling. In (b), the delay times at each step are marked by the vertical lines. The initial distribution function is normal with $\mathcal{P}^{0}(\omega \mid \varnothing)=N\left(0, \sigma^{2}\right)$ and width $\sigma=2 \pi /(90 \mathrm{~ns})$; dephasing is absent. (c) The expected information gain per step (expressed in trits) in the standard Fourier procedure at each of the steps in the continuous case with no dephasing. The dashed red line indicates the maximum possible information gain of 1 trit. The information gain on the seventh step is 0.99 trits. (d) Expected information gain in the second step of the standard (in blue) and modified (with alternative preparation step, in red) Fourier procedures for a continuously distributed field with no dephasing processes.

large in comparison with the saturation time $T_{s}$, the field distribution function becomes periodic and hence semidiscrete; see Fig. 6(a). These oscillations are reflected in the behavior of the information gain of the second step [see Fig. 6(b)]: Although it continues to be a saturation curve, it now exhibits oscillatory peaks which we previously classified as of the third type; the largest of such peaks corresponds exactly to delay time $t_{2}^{F}=t_{1}^{F} / 3$. In the subsequent steps, with the field distribution becoming increasingly more discrete-type, the information gain per step tends asymptotically to the maximum of 1 trit; see Figs. 6(b) and 6(c).

We have previously learned that in the first step, the balanced initial state used in the standard Fourier procedure is inferior as compared with choosing a state from $\mathcal{H}_{X Y}^{3}$. Given this fact, it is interesting to check whether the standard Fourier procedure could be improved through a modification of the preparation stage at each step. We consider the following modification of the standard procedure:

P' In the $i$ th step, the qutrit is prepared in the state $\left|\psi_{(i)}^{0}\right\rangle=\left(\frac{1}{2}|0\rangle+\frac{e^{i \alpha_{i}}}{\sqrt{2}}|1\rangle+\frac{e^{2 i \alpha_{i}}}{2}|2\rangle\right) \in \mathcal{H}_{X Y}^{3}$, where $\alpha_{i}=$ $-\frac{2 \pi}{3}\left(\frac{\xi_{i-1}}{3^{1}}+\frac{\xi_{i-2}}{3^{2}}+\cdots+\frac{\xi_{1}}{3^{i-1}}\right)$ and $\xi_{j}$ is the outcome of the $j$ th step $\left(\alpha_{1}=0\right)$.

E The system is exposed to the magnetic field during time $t_{i}^{F}=t_{1}^{F} / 3^{i-1}$.
R The qutrit is subjected to a Fourier transform $\hat{F}_{3}$ as given by Eq. (6). The information about the field value is extracted through the single-shot projective measurement of the final state in the computational basis. The probability distribution is updated in accordance with Bayes's formula.

Figure 6(d) displays the information gain in the second step for the two cases of standard (shown in blue) and modified (shown in red) Fourier procedure. It turns out that although the modified algorithm produces a higher saturation level and the conditional preparation allows us to extract information above the plateau, after the first step the standard procedure becomes more efficient as the peaks are larger in this case. One should note, though, that this improved efficiency of the standard algorithm can only be exploited with very precisely chosen delay times; otherwise, if the time does not comply with the protocol, the information would be extracted from the plateau level, which is higher in the case of the modified procedure. Note also that the outlined modified procedure is not the same as the one proposed in the main text.

\section{APPENDIX H: LAMA OPERATION}

Shown in Figs. 7(a), 7(c), and 7(e) are the numerically simulated probability distributions for the magnetic field for 

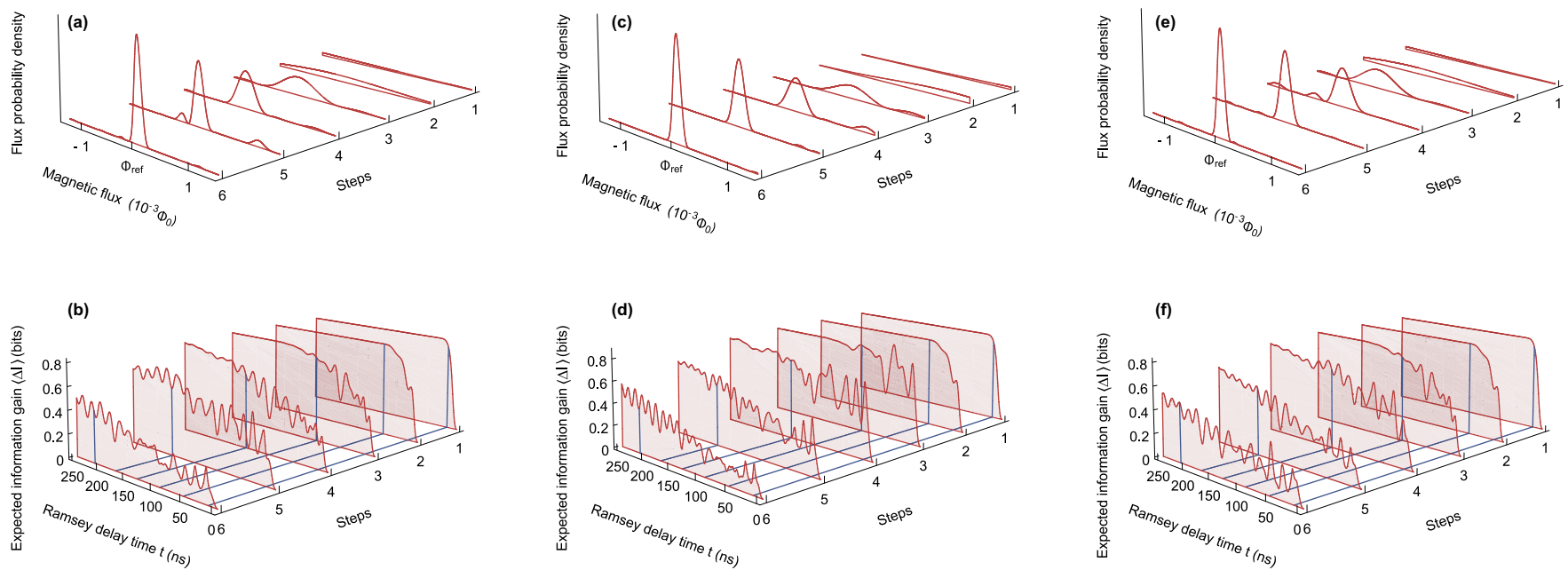

FIG. 7. LAMA operation. (a), (c), and (e) Field distributions and (b), (d), and (f), expected information gains at six different steps of LAMA obtained through numerical modeling in the absence of dephasing. The corresponding outcome sets $\left(\left\{\xi_{1}, \xi_{2}, \ldots, \xi_{6}\right\}\right)$ are (a) and (b) $\{0,2,0,1,1,2\}$, (c) and (d) $\{1,1,1,1,1,1\}$, and (e) and (f) $\{1,2,0,0,1,2\}$. The initial distribution function is $\mathcal{P}^{0}(\omega \mid \varnothing)=N\left(0, \sigma^{2}\right)$ with $\sigma=2 \pi /(90 \mathrm{~ns})$.

different six-step procedures; Figs. 7(b), 7(d), and 7(f) display three corresponding series of expected information gains before the next measurement. The plots correspond to different outcome sets (see Fig. 7 caption) and illustrate the appearance of oscillations in the second and later steps due to the nonzero mean value of the updated distribution.
[1] V. Giovannetti, S. Lloyd, and L. Maccone, Advances in quantum metrology, Nat. Photonics 5, 222 (2011).

[2] G. B. Lesovik, M. V. Suslov, and G. Blatter, Quantum counting algorithm and its application in mesoscopic physics, Phys. Rev. A 82, 012316 (2010).

[3] M. V. Suslov, G. B. Lesovik, and G. Blatter, Quantum abacus for counting and factorizing numbers, Phys. Rev. A 83, 052317 (2011).

[4] C. L. Degen, F. Reinhard, and P. Cappellaro, Quantum sensing, Rev. Mod. Phys. 89, 035002 (2017).

[5] J. Zopes and C.L. Degen, Reconstruction-Free Quantum Sensing of Arbitrary Waveforms, Phys. Rev. Appl. 12, 054028 (2019).

[6] P. Sekatski, M. Skotiniotis, J. Kołodyński, and W. Dür, Quantum metrology with full and fast quantum control, Quantum 1, 27 (2017).

[7] V. V. Zemlyanov, N. S. Kirsanov, M. R. Perelshtein, D. I. Lykov, O. V. Misochko, M. V. Lebedev, V. M. Vinokur, and G. B. Lesovik, Phase estimation algorithm for the multibeam optical metrology, Sci. Rep. 10, 8715 (2020).

[8] S. Danilin, A. V. Lebedev, A. Vepsäläinen, G. B. Lesovik, G. Blatter, and G. S. Paraoanu, Quantum-enhanced magnetometry by phase estimation algorithms with a single artificial atom, npj Quantum Inf. 4, 29 (2018).

[9] A. R. Shlyakhov, V. V. Zemlyanov, M. V. Suslov, A. V. Lebedev, G. S. Paraoanu, G. B. Lesovik, and G. Blatter, Quantum metrology with a transmon qutrit, Phys. Rev. A 97, 022115 (2018).

[10] V. Giovannetti, S. Lloyd, and L. Maccone, Quantum-enhanced measurements: Beating the standard quantum limit, Science 306, 1330 (2004).
[11] L. Vaidman and Z. Mitrani, Qubits Versus Bits for Measuring an Integral of a Classical Field, Phys. Rev. Lett. 92, 217902 (2004).

[12] G. Giedke, J. M. Taylor, D. D’Alessandro, M. D. Lukin, and A. Imamoğlu, Quantum measurement of a mesoscopic spin ensemble, Phys. Rev. A 74, 032316 (2006).

[13] R. S. Said, D. W. Berry, and J. Twamley, Nanoscale magnetometry using a single-spin system in diamond, Phys. Rev. B 83, 125410 (2011).

[14] B. L. Higgins, D. W. Berry, S. D. Bartlett, H. M. Wiseman, and G. J. Pryde, Entanglement-free Heisenberg-limited phase estimation, Nature (London) 450, 393 (2007).

[15] G. Waldherr, J. Beck, P. Neumann, R. S. Said, M. Nitsche, M. L. Markham, D. J. Twitchen, J. Twamley, F. Jelezko, and J. Wrachtrup, High-dynamic-range magnetometry with a single nuclear spin in diamond, Nat. Nanotechnol. 7, 105 (2012).

[16] D. Budker and M. Romalis, Optical magnetometry, Nat. Phys. 3, 227 (2007).

[17] A. Yu. Kitaev, Quantum measurements and the Abelian stabilizer problem, arXiv:quant-ph/9511026 [quant-ph].

[18] R. Cleve, A. Ekert, C. Macchiavello, and M. Mosca, Quantum algorithms revisited, Proc. R. Soc. London, Ser. A 454, 339 (1998).

[19] M. A. Nielsen and I. L. Chuang, Quantum Computation and Quantum Information: 10th Anniversary Edition (Cambridge University Press, Cambridge, 2010).

[20] J. Koch, T. M. Yu, J. Gambetta, A. A. Houck, D. I. Schuster, J. Majer, A. Blais, M. H. Devoret, S. M. Girvin, and R. J. Schoelkopf, Charge-insensitive qubit design derived from the Cooper pair box, Phys. Rev. A 76, 042319 (2007).

[21] E. Il'ichev and Ya. S. Greenberg, Flux qubit as a sensor of magnetic flux, Europhys. Lett. 77, 58005 (2007). 
[22] M. Bal, C. Deng, J.-L. Orgiazzi, F. R. Ong, and A. Lupascu, Ultrasensitive magnetic field detection using a single artificial atom, Nat. Commun. 3, 1324 (2012).

[23] W. Wang, Y. Wu, Y. Ma, W. Cai, L. Hu, X. Mu, Y. Xu, ZiJie Chen, H. Wang, Y. P. Song, H. Yuan, C.-L. Zou, L.-M. Duan, and L. Sun, Heisenberg-limited single-mode quantum metrology in a superconducting circuit, Nat. Commun. 10, 4382 (2019).

[24] A. A. Abdumalikov Jr., J. M. Fink, K. Juliusson, M. Pechal, S. Berger, A. Wallraff, and S. Filipp, Experimental realization of non-Abelian non-adiabatic geometric gates, Nature (London) 496, 482 (2013).

[25] K. S. Kumar, A. Vepsäläinen, S. Danilin, and G. S. Paraoanu, Stimulated Raman adiabatic passage in a three-level superconducting circuit, Nat. Commun. 7, 10628 (2016).
[26] P. Magnard, P. Kurpiers, B. Royer, T. Walter, J.-C. Besse, S. Gasparinetti, M. Pechal, J. Heinsoo, S. Storz, A. Blais, and A. Wallraff, Fast and Unconditional All-Microwave Reset of a Superconducting Qubit, Phys. Rev. Lett. 121, 060502 (2018).

[27] T. Elo, T. S. Abhilash, M. R. Perelshtein, I. Lilja, E. V. Korostylev, and P. J. Hakonen, Broadband lumped-element Josephson parametric amplifier with single-step lithography, Appl. Phys. Lett. 114, 152601 (2019).

[28] M. J. Peterer, S. J. Bader, X. Jin, F. Yan, A. Kamal, T. J. Gudmundsen, P. J. Leek, T. P. Orlando, W. D. Oliver, and S. Gustavsson, Coherence and Decay of Higher Energy Levels of a Superconducting Transmon Qubit, Phys. Rev. Lett. 114, 010501 (2015).

[29] H.-P. Breuer and F. Petruccione, The Theory of Open Quantum Systems (Oxford University Press, New York, 2007). 